\title{
Research on Application System of Three-Dimensional Design of Transmission Line Based on Grid GIS Cloud Platform
}

\author{
Xinmin $\mathrm{Yu}^{1}$, Tianrong Zhong ${ }^{2 *}$, Xianri Wang ${ }^{1}, \mathrm{Kejian} \mathrm{Nie}^{1}$, and Jinglei $\mathrm{Lv}^{2}$ \\ ${ }^{1}$ Research Institute of Economics and Technology of Fujian Electric Power Co., Ltd., Fuzhou, Fujian, 350001, China \\ ${ }^{2}$ Xiamen Great Power Geo Information Technology Co. Ltd., Xiamen, Fujian, 351008, China
}

\begin{abstract}
Intelligent integration has been basically achieved in transmission line design in developed countries, while the current management design level in this field in China is backward and inefficient. According to the characteristics of the pilot transmission line project, it is necessary to build a transmission line 3D design application system based on the grid GIS cloud platform with the advantages of webgl cross platform and without installing plug-ins, to assist in the application of transmission line 3D design, and to develop the interface with professional line 3D design software, so as to realize the smooth operation of GIS elevation and image information and other information such as line selection and capital collection. Experiments prove that the construction of transmission line three-dimensional design application system based on grid GIS cloud platform provides a set of simple, fast, convenient and intelligent and efficient three-dimensional design support for planners and designers. It is an effective means for modern power enterprises to improve management design level and work efficiency, and has great significance in economic and social benefits.
\end{abstract}

\section{Introduction}

Development and Construction of Grid GIS Cloud Platform within the National Grid, business covers power grid production, marketing, planning, construction, dispatching, materials, communications, emergency and other important business of power grid. Grid GIS Cloud Platform provides six types of integration, an open and enterprise-oriented public service platform for grid spatial information has been formed. It provides technical support for the application of production, marketing, planning and other professional fields, and has been integrated with 13 integrated business systems, such as production management, marketing management, planning and design, distribution automation, transmission and transformation condition monitoring.

With the Constant Construction of Strong Smart Grid, in order to better manage the increasing amount of temporal power grid data, better support for professional visualization applications based on geographic information, Grid GIS Cloud Platform Begins in 2015. After the first phase of research and development, it realizes the transformation from traditional IT architecture to cloud platform architecture, and builds a grid cloud GIS platform with high performance, high reliability, easy scalability, perfect function, simple and easy to use characteristics with cloud GIS as the core. In the first phase of design and development, it has completed the development of cloud GIS resource management center, cloud computing center, GIS data cloud storage center and other functional modules. The first phase of grid GIS cloud platform has been deployed in Fujian, Shanghai, Hunan, Henan and Zhejiang provinces. Through trial operation the advancement, practicability and stability of cloud-based GIS platform are preliminarily verified. At present, the platform has a stable and efficient GIS core and general services, can manage massive temporal spatial two-dimensional data, and provide a variety of SDK support Web and PC end secondary development system. The second phase of grid GIS cloud platform has extended the scope of implementation, further strengthen the application support of three-dimensional modeling, two-dimensional and three-dimensional integration and mobile GIS client.

At present, Three-dimensional Geographic Information System (3DGIS) has been more and more widely used in the whole power industry. The application of 3D GIS technology to the 3D design of power grid lines has become an effective means for modern power enterprises to improve their management design level and work efficiency by providing a simple, rapid, convenient, intelligent and efficient 3D design support for planners and designers.

\section{Necessity}

Transmission line is the physical channel of electric power transmission, and it is also an important carrier of power communication guarantee at the same time. It has the characteristics of wide geographical distribution, complex operation conditions, vulnerability to natural

*Corresponding author's e-mail: ztr203@163.com 
environmental impact and external damage, and heavy maintenance workload ${ }^{[1]}$. Survey digitization and design visualization are the concrete embodiment of the grid line intellectualization requirements in the survey design process. It has great significance to implement the intellectualization requirements from the source of the line engineering and to build the entire line engineering according to the intellectualized goals. Survey digitization is the use of satellite images, aerial photographs and satellite positioning and other means, and construction of Digital Three-Dimensional Geographic Information System. It realizes digitization of line survey, path optimization, pole and tower positioning and so on by avoiding the bad weather and geological conditions that affect the safety of transmission lines, and improves line disaster resistance.

Data is one of productivity ${ }^{[2]}$. Establishing interactive database, it can effectively accumulate and manage power resource data in the region. The rich and valuable information resources can be formed through long-term and continuous data accumulation, and the accumulation of information resources is an important basis for the core competence of survey and design market. It is also beneficial to deepen the depth and breadth of power survey and design research, and improve the design efficiency.

Transmission line design in developed countries has basically achieved intelligent integration, but China is still a blank in this field. Through the research of transmission line aided design platform based on threedimensional visualization, it is bound to speed up the design and construction progress of transmission line projects. In line with the guiding ideology of the State Grid to focus on "resource conservation, environmental friendliness" and the construction of "two types, three new" transmission and transformation projects. It has great significance in economic and social benefits.

The application platform for three-dimensional design of transmission lines can ensure that the elevation and image information of geographic information system and other information such as line selection and collection can be smoothly imported into the professional three-dimensional design software of transmission lines, and provide a set of simple, fast, convenient, intelligent and efficient three-dimensional design support for planners and designers. It is an effective means for modern electric power enterprises to improve management design level and work efficiencyt and it is of great significance in economic and social benefits.

\section{Key Technologies}

\subsection{Multilevel LOD Processing Technology for Three-Dimensional Design Data of Transmission Lines}

LOD is Levels of Detail, which means multi-level details. It refers to a computer graphics strategy to reduce the complexity of a scene based on the fact that the 3D object is far from the viewpoint or other conditions that do not affect the visual effect of the picture. The purpose of LOD modeling is to obtain the best rendering effect and meet the needs of smooth and smooth interactive roaming. Reasonable level of LOD model is the key problem to be solved ${ }^{[3]}$.

The relationship between the visual effect and the amount of model data must be weighed for the rendering of three-dimensional large scene model of transmission line design. Due to the limitation of memory size and application memory addressing space, the scheduling scope of large-scale fine three-dimensional model is smaller. To break through the memory limitation of large scene scheduling, we need to compress and simplify the finest three-dimensional model to produce multi-level LOD model. By optimizing the scheduling algorithm, rendering index is created for three-dimensional scene, and different LOD levels are set for the model and texture of elements. When roaming, different scene model texture can be loaded according to the current camera perspective under the minimum resource occupation, so that the whole roaming process is smoother.

\subsection{Dynamic dispatching mechanism of 3D design data for transmission lines}

At present, the computer can not load massive data at one time, so dynamic scheduling technology must be used to realize roaming and application of large-scale three-dimensional design scenarios of transmission lines. Massive data dynamic mobilization technology mainly uses multi-level LOD technology, cone sorting technology, data paging, caching technology and so on ${ }^{[4]}$

Through optimization of dispatching algorithm for three-dimensional design of transmission line, rendering index is created for three-dimensional scene. Different LOD levels are set for the model and texture of elements. When roaming dispatch, different scene model texture can be loaded according to the current camera perspective under the minimum resource occupation, so that the whole roaming process is smoother.

\subsection{Three dimensional data simplification technology of transmission line three dimensional design}

Modeling simplification technology is an automatic hierarchical compression algorithm for triangular surface number, texture mapping number and texture resolution of three-dimensional model without significant distortion [5].

Simplification of models is an important and effective method to speed up the visualization of threedimensional scenes in transmission line design, but the simplification of models can easily produce visual mutations, which greatly affects the visual effect. Threedimensional geometric models are usually represented by polygonal meshes. Model simplification does not simply reduce the number of triangular patches in the mesh model, but correspondingly reduces the number of triangular patches in the model according to the requirement of approximation accuracy of the original 
model. Therefore, it is necessary to delete those triangles which have little influence on the model and retain those which can reflect the geometric characteristics of the model.

\subsection{Tile Processing Technology for Three- Dimensional Design Data of Transmission Lines}

Pyramid model is a multi-resolution Hierarchy model, in a sense, it can be said to be a continuous multi-resolution hierarchical model ${ }^{[6]}$. It usually constructs pyramids by multiplier method to form multiple resolution levels. From the bottom to the top of the pyramid, the resolution becomes lower and lower, but the range of representation remains unchanged. By constructing pyramid model, three-dimensional data with different accuracy can be provided for three-dimensional visualization system. Combining three-dimensional data of different accuracy with pyramid model, loading data dynamically in real time can reduce memory consumption and enable client to realize visualization of large-scale transmission line three-dimensional design data.

Octree indexing mechanism is a spatial indexing mechanism of three-dimensional tile data, which is similar to terrain image data. Quadtree method is extended to three-dimensional, and octree spatial indexing mechanism of three-dimensional tile data is established. The basic idea of octree data structure is to divide three-dimensional regions into three-dimensional grids. Each small cube (called a volume element) has one or more attribute data. Areas with the same attributes are represented by large blocks, while complex areas are represented by small blocks. When large blocks are divided into small blocks, they are divided into eight rules ${ }^{[7]}$.

Three-dimensional tile technology supports multilayer pyramid and efficient indexing mechanism, so it is no longer limited by the original accuracy and size of transmission line three-dimensional design data, and accelerates the loading and rendering speed of massive three-dimensional model data. Three-dimensional tile technology mainly adopts pyramid indexing mechanism of quadtree structure and octree structure ${ }^{[8]}$.

\subsection{Application of Lightweight Visualization Technology in Three-Dimensional Design of Transmission Lines}

The lightweight visualization of massive DSM data in the network environment is one of the front issues in the current network 3D GIS research. Aiming at the problems of low efficiency and poor interaction of massive three-dimensional model data network in transmission line design, a lightweight network visualization method based on massive DSM data is adopted ${ }^{[9]}$. According to the characteristics of WebGL technology, by optimizing the organization and management of massive DSM data, adaptive selection of LODs and dynamic scheduling and other key technologies, using HTML5 to display three-dimensional data pages, the application of transmission line three- dimensional design is realized. The system has the advantages of plug-in-free, cross-platform, openness and so on ${ }^{[10]}$.

\section{Thought of System Implementation}

The three-dimensional design application system of transmission line follows the construction idea of "big platform, micro application, multi-scene, component" of grid GIS cloud platform [11], The basic platform is supported by the grid GIS cloud platform, and the public data and functional service components are provided by the platform. According to the business and the individual needs of Fujian transmission line project, customizable components can be customized. According to the secondary development interface of the basic platform, new business micro-applications can be reasonably organized to meet the requirements of twodimensional and three-dimensional GIS application for transmission line design in an all-round way.

\subsection{Specification for Data Base Construction of Transmission Line Design Achievements}

According to the characteristics of digital design data of transmission line engineering, layered superposition of geographic entity elements according to unified threedimensional model geographic coordinates is the basic idea of data organization. According to the characteristics of basic spatial data, three-dimensional spatial data is constructed according to the hierarchical framework of "database-element class-layer-element and attribute". Data are aggregated according to the principle of classifying and layering according to the characteristics of digital design data of transmission line engineering. At the same time, in order to achieve better management and use of data, data is mainly classified according to content in data hierarchy. In terms of elements and attributes, the principle is that each element only stores attributes, spatial index fields, annotated fields and uniquely identifiable associated fields. The basic attribute fields are not directly stored in the attribute table of the map layer, but are related fields with uniquely identifiable fields.

According to the actual needs of business departments to retrieve power grid equipment, in order to facilitate users'habits, the three-dimensional model data are logically classified and organized, the navigation tree of power grid equipment is established, and the data is organized and transferred in a tree structure.

\subsection{Construction of Basic Geographic Information Database}

Through three-dimensional data processing and management tools, image data processing tools, terrain data processing tools, vector data processing tools, etc., high-precision image, elevation data, power grid data, channel data, thematic data and other data processing are realized. At the same time, relevant data are stored and managed structurally and unstructured. 


\subsection{Construction of three-dimensional database of power grid}

According to the data provided by the digitization of transmission line project, through the three-dimensional data processing and management tools, BIM/GIM data analysis and processing tools, tilt photography data processing tools, three-dimensional laser point cloud data processing tools, three-dimensional model data processing tools, etc., the BIM/GIM data file analysis, structured data extraction, tilt photography data, threedimensional point cloud number are realized. Threedimensional tile processing of data and threedimensional model data, and storage and management of structured and unstructured data.

\subsection{Construction and management of three- dimensional design resource database for transmission lines}

Based on the three-dimensional series of standards, it can realize the analysis of the general model and product model of three-dimensional design, extract the threedimensional standard model data including engineering information, form the three-dimensional design resource database of transmission line, display the model and the affiliated structured information, provide the functions of model base management and scene configuration through the three-dimensional data processing and management tools, and realize the different levels of threedimensional model. The management of digitized achievements provides a basis for the follow-up application of progress, investment, safety, quality control and so on.

\subsection{Two-dimensional and three-dimensional integrated scene construction}

Integrating image service, elevation service, vector service, navigation service, grid equipment graphics service, BIM/GIM data, image data, elevation data, vector data, tilt photography data, three-dimensional point cloud data, three-dimensional model data, grid data, channel data, thematic data and other massive data, according to transmission line design project. With the requirement of business scenario, customize twodimensional and three-dimensional integrated application scenarios to meet different business requirements of transmission line design engineering.

\subsection{Adaptability Adjustment of Two- dimensional and Three-dimensional Integrated Platform for Power Grid GIS}

According to the three-dimensional design business characteristics of transmission lines, the two-dimensional and three-dimensional data processing and management tools of power grid GIS are adaptively adjusted, and the functions of BIM/GIM data, image data, elevation data, vector data, tilt photography data, three-dimensional point cloud data, three-dimensional model data, power grid data, channel data, thematic data, model database management and scene configuration are provided. It can meet the requirements of two-dimensional and threedimensional data processing in transmission line design.

According to the three-dimensional design business characteristics of transmission lines, the two-dimensional and three-dimensional service publishing platform of power grid GIS can be adaptively adjusted to publish BIM/GIM data, image data, elevation data, vector data, tilt data, three-dimensional point cloud data, power grid data, channel data, thematic data and other data services. The functions of two-dimensional and three-dimensional data positioning, query and analysis can be realized to meet the requirements of transmission line design. Consider the requirements of two-dimensional and threedimensional data sharing.

\subsection{Development and Implementation of Transmission Line 3D Design Application System Based on Grid GIS Cloud Platform}

According to the characteristics of transmission line design business, JSON and Ajax are used to organize and transmit data, HTML5 and WebGL technology are used to display three-dimensional data, which has the advantages of plug-in-free, cross-platform and openness. The basic application framework of transmission line three-dimensional design is realized. On the basis of the application framework, plug-in mode is used to realize three-dimensional data management, three-dimensional design data processing and transmission line data display. Business micro-applications, such as visualization display of engineering, view of Engineering attribute information, display of transmission line engineering tree, full-text retrieval of transmission line engineering, display of three-dimensional design general model, etc., are customized to develop two-dimensional and threedimensional support applications for transmission line design, and realize the full application of threedimensional design results in the process of Engineering construction.

\subsection{Software Integration of Professional Line Three-Dimensional Design}

The application system of transmission line threedimensional design based on grid GIS cloud platform integrates with professional line three-dimensional design software and interface integration with standard web services.

\section{Summary}

By establishing the application system of transmission line three-dimensional design based on grid GIS cloud platform, the transformation of design mode from traditional two-dimensional mode to three-dimensional visualization mode is realized ${ }^{[12]}$. Combing the application process and business requirements of transmission line three-dimensional design, building a three-dimensional transmission line design application 
technology framework including geographic information technology, three-dimensional modeling technology, three-dimensional visualization technology and other key technologies. The application system of threedimensional design and design of transmission lines based on geographic information system, threedimensional model and three-dimensional visualization is established. Establishment of three-dimensional design and application technology route of transmission line based on basic geographic information data and higher precision survey data. Combing the application results of three-dimensional design of transmission lines, formulating the data types and chart formats of the design application results, formulating the format specifications of the design results documents, so as to standardize and standardize the application workflow of three-dimensional design of transmission lines. By discussing and researching the implementation scheme of the three-dimensional design application platform for transmission lines, the logical framework, data framework and functional framework of the threedimensional design application platform for transmission lines are established, and the overall implementation scheme is formulated. The contents of data storage information, data types and calling conditions are sorted out and the database of design resources is constructed in the three-dimensional design of transmission lines. According to the characteristics of Fujian transmission line, the application platform for three-dimensional design of transmission line is constructed. According to the pilot project, the application software for threedimensional design of transmission line is tried out to assist in the application of three-dimensional design of transmission line, and the interface with professional three-dimensional design software for transmission line is developed to ensure the smooth introduction of elevation and image information of GIS, other information of line selection and collection, and to realize three-dimensional design and collaborative work. Work and resource sharing can effectively improve the management design level and work efficiency of enterprises ${ }^{[13]}$.

\section{References}

1. Zeng Qingpei. Research on conductor galloping of high voltage transmission lines [D]. Tianjin University, 2012.

2. Feng Lixin, Sun Yong. Big data is also a kind of productivity - Interview with Li Poxi, Vice President of China Productivity Society [J]. Enterprise Reform and Management, 2014 (9): 1-1.

3. Tang Guiwen, Chen Xuexia, Ren Chao. Research on Key Technologies of View-Dependent LOD Quadtree Algorithms in Virtual Reality Applications [J]. Computer Engineering and Design, 2008, 29 (4): 928-930.

4. Hu Bin, Jiangnan, Liu Chen, et al. Research on the dynamic dispatching mechanism of large terrain based on coarse-grained paging and fine-grained fragmentation [J]. Computer Applications, 2006, 26 (b06): 3-4.

5. Feng Xiang, Zhou Mingquan. Simplification algorithm of three-dimensional model with texture [J]. Journal of Computer Aided Design and Graphics, 2009, 21 (6): 842-846.

6. Liu Baohong. Research on Theory and Key Technologies of Multiresolution Modeling [D]. National University of Defense Science and Technology, 2003.

7. Zhang Yongyu, Ma Jinsong. Research on the Establishment and Query Algorithms of Linear Octree Spatial Index in 3D GIS [J].Computer Engineering and Science, 2009, 31(2): 61-63.

8. Yu Huaqiang, Liu Bing, Shi Zejie, et al. Making of three-dimensional tile map and its two-dimensional and three-dimensional coordinate transformation [J].Surveying and Spatial Geographic Information, 2017 (9). 139-142.

9. Zhang Yunhao, Zhu Qing, Zhu Jun, et al. Network Lightweight Visualization Method for Massive DSM Data [J]. Journal of Surveying and Mapping Science and Technology, 2017, 34 (6). 649-653.

10. Wei Shuhan, Sun Qi. Design and research of threedimensional intelligent community management system based on HTML5 and WebGL [J].Industrial Control Computer, 2017(5). 139-140.

11. Fujian Electric Power Company of State Grid fully utilizes the instant communication platform to support the command work against typhoon [J]. Electric Power Information and Communication Technology, 2016 (7): 118-118.

12. Fan Liangyi.Interior design method based on BIM and its application[J]. China Architectural Decoration and Decoration, 2017(09): 112-115.

13. LI Tie-ding, LIU Wenxun, LIN Fang, et al. Application and Prospect of the Whole Process of Digital Design for Transmission Line Engineering [J]. Smart Grid, 2016, 4 (3): 323-327. 